\title{
A Spectrum Detection Approach for Bearing Fault Signal Based on Spectral Kurtosis
}

\author{
Yunfeng Li, Liqin Wang, and Jian Guan \\ School of Mechanical Engineering, Harbin Institute of Technology, Harbin, China \\ Correspondence should be addressed to Liqin Wang; lqwang@hit.edu.cn
}

Received 2 August 2016; Revised 9 November 2016; Accepted 4 December 2016; Published 19 February 2017

Academic Editor: Angelo M. Tusset

Copyright ( 2017 Yunfeng Li et al. This is an open access article distributed under the Creative Commons Attribution License, which permits unrestricted use, distribution, and reproduction in any medium, provided the original work is properly cited.

\begin{abstract}
According to the similarity between Morlet wavelet and fault signal and the sensitive characteristics of spectral kurtosis for the impact signal, a new wavelet spectrum detection approach based on spectral kurtosis for bearing fault signal is proposed. This method decreased the band-pass filter range and reduced the wavelet window width significantly. As a consequence, the bearing fault signal was detected adaptively, and time-frequency characteristics of the fault signal can be extracted accurately. The validity of this method was verified by the identifications of simulated shock signal and test bearing fault signal. The method provides a new understanding of wavelet spectrum detection based on spectral kurtosis for rolling element bearing fault signal.
\end{abstract}

\section{Introduction}

The rolling element bearing is one of the most important parts in aircraft engines. Many bearing incidents in aircraft engines have indicated that the initial damage of bearing occurred on the surface and subsurface. This initial damage appears as the peeling and develops into spalls eventually [1]. During the performance and reliability testing, the vibration signal can reveal the failure state in time and frequency domain promptly. In order to obtain the fault characteristic parameters of the rolling bearing, a variety of signal processing methods are applied to process the rolling bearing vibration signal. Since wavelet transform has the property of time-frequency localization, it is utilized to detect the signal transients from the raw signal extensively [2-6]. The wavelet transform provides an analysis method both in time domain and in frequency domain, which becomes an important method in the nonstationary signal analysis. It is well known that the similarity exists between Morlet wavelet and the impact response of the attenuation components, and the Morlet wavelet is Gaussian in the frequency domain. In addition to the above factors, the time-frequency structure of the Morlet wavelet is optimal match with the typical transients. Therefore, the Morlet wavelet is more suitable for the extraction of rotating machinery fault signal and the rolling bearing fault diagnosis.
The purpose of wavelet transform is to select the proper frequency band of the correct faulty information and the best filter. Therefore, it is necessary and urgent to select the reasonable resonance frequency band for the demodulation technology. At the same time, an index unaffected by experimental conditions is needed to measure the transform result. Kurtosis is a normalized time domain statistics parameter, which is sensitive to the instantaneous signal. Thus, the spectral kurtosis (SK) is an effective tool to get the fault signals of rolling bearing and the reasonable bandwidth $[7,8]$. SK is used to analyze the Gaussian components of the signal and locate these components in the frequency domain. It can be seen as a measurement of the energy distribution at each frequency. The peeling of the faulty bearing produces a nongauss distribution signal, which can be described by the time averaging statistic. The final purpose of SK is to detect the fault signal characteristics in the filtering results [9-11].

The concept of SK was proposed by Dwyer firstly [12]. Then, it was successfully applied to the rolling bearing resonance peak and resonance band selection by Antoni and Randall [13]. Fast Kurtogram based on band-pass filter and short-time Fourier transform (STFT) has made SK a practical tool for the fault diagnosis [14]. Nevertheless, in order to obtain a maximum value of the spectral kurtosis, the STFT window must be shorter than the spacing between the pulses and longer than the individual pulses. The maximum spectral 
kurtosis value corresponding to the band is not always the optimal band by the above method. In order to solve these problems, various kinds of improved algorithms based on spectral kurtosis and other signal processing technologies were presented, such as searching all the possible resonance frequency bands adaptively by shifting and expanding predetermined Morlet wavelet [15].

In this paper, the Morlet wavelet and SK are combined. The spline curve function is utilized to obtain the optimal wavelet filter range, and different wavelet clusters are designed to detect the impact frequency. Firstly, a group of Morlet wavelet cluster with different quality factor $Q$ is designed and the spectral kurtosis of the filtered signal is calculated, respectively. Then, the trend of spectral kurtosis is drawn by the spline curve based on a limited number of points. The signal detection range and filter bandwidth are gradually reduced. As a consequence, the accuracy of fault diagnosis is improved by the optimized filter.

\section{Adaptive Spectral Kurtosis Filtering Based on Morlet Wavelet}

2.1. Morlet Wavelet. The complex Morlet wavelet is the product of a complex exponential function multiplied by a Gaussian function. The expression of Morlet wavelet is

$$
\operatorname{Morl}(t)=\frac{\sigma}{\sqrt{\pi}} e^{-\sigma^{2} t^{2}} e^{i 2 \pi f_{0} t}
$$

Meanwhile, the shape of Gaussian window in the frequency domain can be derived as [16]

$$
\operatorname{Morl}(f)=\operatorname{Morl}^{*}(f)=e^{-\left(\pi^{2} / \sigma^{2}\right)\left(f-f_{0}\right)^{2}},
$$

where $\operatorname{Morl}(f)$ is the fast Fourier transformation of $\operatorname{Morl}(t)$. Since $\operatorname{Morl}(f)$ is real, $\operatorname{Morl}(f)=\operatorname{Morl}^{*}(f)$, and the superscript $*$ denotes the complex conjugate. $f_{0}$ is the center frequency of the wave and $\sigma$ is waveform parameter that determines its width. For Morlet wavelet, the center frequency $f_{0}$, and shape factor $\sigma$ affect the shape and location simultaneously. By (1), the corresponding wave shape of Morlet wavelet is shown in Figure 1.

In Figure 1, Morl1 is the wave of the Gaussian function, and Morl2 is the complex exponential wave function. They are expressed as

$$
\begin{aligned}
& \text { Morl1 }=e^{-t^{2} \sigma^{2}}, \\
& \text { Morl2 }=e^{i 2 \pi f_{0} t} .
\end{aligned}
$$

According to the Euler formula, Morl2 can be expressed as Morl2 $=\cos \left(2 \pi f_{0} t\right)+i \sin \left(2 \pi f_{0} t\right)$, where $\operatorname{Re}(\operatorname{Mor} 2)=$ $\cos \left(2 \pi f_{0} t\right)$. If $t_{1}$ is half a cycle of Morll and $t_{2}$ is a quarter period of $\operatorname{Re}\left(\right.$ Morl2), $t_{1}$ and $t_{2}$ can be deduced as

$$
\begin{aligned}
& t_{1}=\frac{\sqrt{\ln m^{-1}}}{\sigma}, \\
& t_{2}=\frac{\arccos (m)}{2 \pi f_{0}},
\end{aligned}
$$

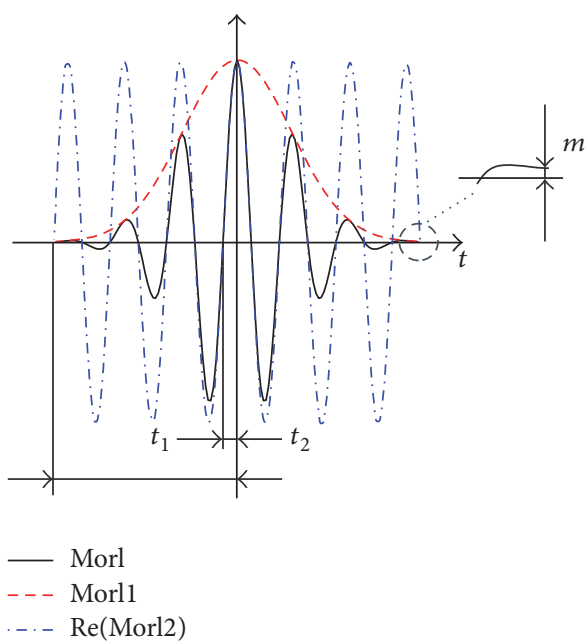

FIGURE 1: Structure of Morlet wavelet.

where $m$ is the value of Morlet wave endpoint and it is infinitesimal (as shown in Figure 1). It is necessary to ensure that the Morlet wavelet is fluctuant enough and compactly supported. For Morl1 and Morl2, the period ratio $T_{1} / T_{2}$ is

$$
\frac{T_{1}}{T_{2}}=\frac{2 t_{1}}{4 t_{2}}=\frac{Q \pi \sqrt{\ln m^{-1}}}{\arccos (m)}=k,
$$

where $Q$ is the quality factor, $Q=f_{0} / \sigma$. When $k_{1} \leq k \leq k_{2}$, Morlet wavelet at most includes $k_{2}$ Morl2 and at least $k_{1}$ Morl2. If (5) is introduced into $k_{1} \leq k \leq k_{2}$, the expression of the quality coefficient $Q$ can be obtained as follows:

$$
\frac{k_{1} \arccos (m)}{\pi \sqrt{\ln m^{-1}}} \leq Q \leq \frac{k_{2} \arccos (m)}{\pi \sqrt{\ln m^{-1}}} .
$$

The quality coefficient $Q$ is proportional to the fluctuation of the Morlet wavelet. The larger the quality coefficient $Q$ is, the smaller the volatility of the Morlet wavelet is and vice versa. Therefore, when $0.5 \leq k \leq 2$ and $m=0.00001$, the value range of $Q$ is $0.0217 \leq Q \leq 0.0869$.

The corresponding family of wavelets consists of a series of son wavelets, which are generated by dilation and translation from the mother wavelet shown as follows:

$$
\operatorname{Morl}_{a, b}(t)=\frac{1}{\sqrt{a}} \operatorname{Morl}\left(\frac{t-b}{a}\right),
$$

where $a$ is the scale factor and $b$ is wavelet displacement factor (the constant $1 / \sqrt{a}$ is used for energy normalization). The wavelet transform coefficient is defined by the following equation:

$$
\begin{aligned}
\mathrm{WT}_{(a, b)} & =\int_{-\infty}^{+\infty} x(t) \cdot \operatorname{Morl}_{a, b}^{*}(t) \cdot d t \\
& =\left\langle x(t), \operatorname{Morl}_{a, b}(t)\right\rangle,
\end{aligned}
$$

where $\operatorname{Morl}_{a, b}^{*}(t)$ is the conjugate complex numbers of $\operatorname{Morl}_{a, b}(t)$ and $\langle\cdot\rangle$ represents solving convolution. 
2.2. Spline Curve Interpolation. Since the SK values are not continuous, the spline curve is used to obtain the variation trend of SK on the basis of discrete points. It can provide the basis for optimizing the analysis range.

The function of spline curve interpolation is an approximation of piecewise three polynomials, but it is continuous. Its first- and second-order derivatives are both continuous. The value of $x_{j}$ is $y_{j}=f\left(x_{j}\right)(j=0,1, \ldots, n)$, spline curve interpolation function is $S(x)$, and its first-order derivative $S^{\prime}\left(x_{j}\right)=m_{j} . S(x)$ can be expressed as

$$
S(x)=\sum_{j=0}^{n}\left[y_{j} \alpha_{j}(x)+m_{j} \beta_{j}(x)\right],
$$

where $\alpha_{j}(x)$ and $\beta_{j}(x)$ are the interpolation function, expressed by

$$
\begin{aligned}
& \alpha_{j}(x) \\
& = \begin{cases}\left(\frac{x-x_{j-1}}{x_{j}-x_{j-1}}\right)^{2}\left(1+2 \frac{x-x_{j}}{x_{j-1}-x_{j}}\right) & x_{j-1} \leq x \leq x_{j} \quad(j \neq 0) \\
\left(\frac{x-x_{j+1}}{x_{j}-x_{j+1}}\right)^{2}\left(1+2 \frac{x-x_{j}}{x_{j+1}-x_{j}}\right) & x_{j} \leq x \leq x_{j+1} \quad \text { Others, } \\
0 & (j \neq n)\end{cases} \\
& \beta_{j}(x)= \begin{cases}\left(\frac{x-x_{j-1}}{x_{j}-x_{j-1}}\right)^{2}\left(x-x_{j}\right) & x_{j-1} \leq x \leq x_{j}(j \neq 0) \\
\left(\frac{x-x_{j+1}}{x_{j}-x_{j+1}}\right)^{2}\left(x-x_{j}\right) & x_{j} \leq x \leq x_{j+1}(j \neq n) \\
0 & \text { Others. }\end{cases}
\end{aligned}
$$

Under the condition of corresponding boundary, the function $S(x)$ can be calculated when the equations of $m_{j}(j=0,1, \ldots, n)$ were obtained [17].

2.3. Transient Signal Detection Based on SK. The nonstationary signal is normally expressed by Wold-Cramér decomposition; that is,

$$
Y(t)=\int_{-\infty}^{+\infty} e^{j 2 \pi f t} H(t, f) d X(f),
$$

where the time-varying transfer function $H(t, f)$ can be interpreted as the complex envelope or complex demodulate of process $Y(t)$ at frequency $f$.

The signal characteristics information in $H(t, f)$ can be described by the spectral moments, the expression of which is

$$
\begin{aligned}
S_{2 n Y}(f) & \triangleq E\left\{S_{2 n Y}(t, f)\right\}=\frac{E\left\{|H(t, f) d X(f)|^{2 n}\right\}}{d f} \\
& =E\left\{|H(t, f)|^{2 n}\right\} \cdot S_{2 n x} .
\end{aligned}
$$

Under the conditions of ergodicity and stationarity of $H(t, f)$, it is easy to prove that

$$
S_{2 n Y}(f)=\left\langle S_{2 n Y}(t, f)\right\rangle_{t} .
$$

The significant property of nonstationary processes is non-Gaussian. For a non-Gaussian process, spectrum accumulation that is greater than or equal to the fourth order is a nonzero value. Therefore, the fourth-order spectral accumulation is adopted, which is defined as

$$
C_{4 Y}(f)=S_{4 Y}(f)-2 S_{2 Y}^{2}(f) .
$$

The greater the degree of signal deviation from Gaussianity is, the greater its fourth-order spectral accumulation is. Therefore, the energy normalized fourth-order spectral accumulation can be used to measure the peak of the signal process probability density at the frequency $f$, that is, spectral kurtosis.

The wavelet coefficients can be obtained by calculating the signal convolution by each row of the filter. The higher value of the wavelet coefficient is, the more similar the wavelet function with the signal waveform will be. The values of the wavelet coefficients are equal to the square of energy. SK is used to measure the energy spectrum. Therefore, SK achieves the maximum value when the waveforms are most similar [16]. Equation (15) gives the value of spectral kurtosis for each filter [18]:

$$
\begin{aligned}
\operatorname{kurtosis}(y) & \triangleq \frac{C_{4 Y}(f)}{S_{2 Y}^{2}(f)}=\frac{S_{4 Y}(f)}{S_{2 Y}^{2}(f)}-2 \\
& =\frac{\operatorname{mean}\left(H^{4}(t, f)\right)}{\left(\text { mean }\left(H^{2}(t, f)\right)^{2}\right.}-2 .
\end{aligned}
$$

2.4. Construction of Wavelet Clusters. Adaptive wavelet method uses spectral kurtosis as the evaluation index of Morlet wavelet filtered results. This method can optimize the latter filter parameters according to the calculation results in the previous step. Each step can improve the calculation accuracy and reduce the searching scope effectively. Compared with other bearing fault diagnosis method based on kurtosis, the accuracy of fault frequency identification and the detection bandwidth are both optimized.

During the construction of octave filter with complex translation Morlet wavelet, the number of filters that cover the entire analysis band is $M=K \times N$, where $K$ is the number of the filters in each octave and $N$ is the number of the octaves [19].

The central frequency of the filter is expressed as

$$
f_{m k}=\frac{f_{\max }-f_{\min }}{\left(2^{1 / K}\right)^{n}} \quad(n=1,2, \ldots, M),
$$

where $\left[f_{\min }, f_{\max }\right]$ is the frequency band range of the bearing characteristic frequencies in the operating mode. Bandwidth of each filter is expressed by

$$
\sigma_{n K}=f_{n K} \cdot \frac{2}{K}
$$

The value of $K$ and $N$ and the frequency analysis range $\left[f_{\min }, f_{\max }\right]$ containing all possible fault frequencies are initially set. The center frequencies and bandwidths can be 


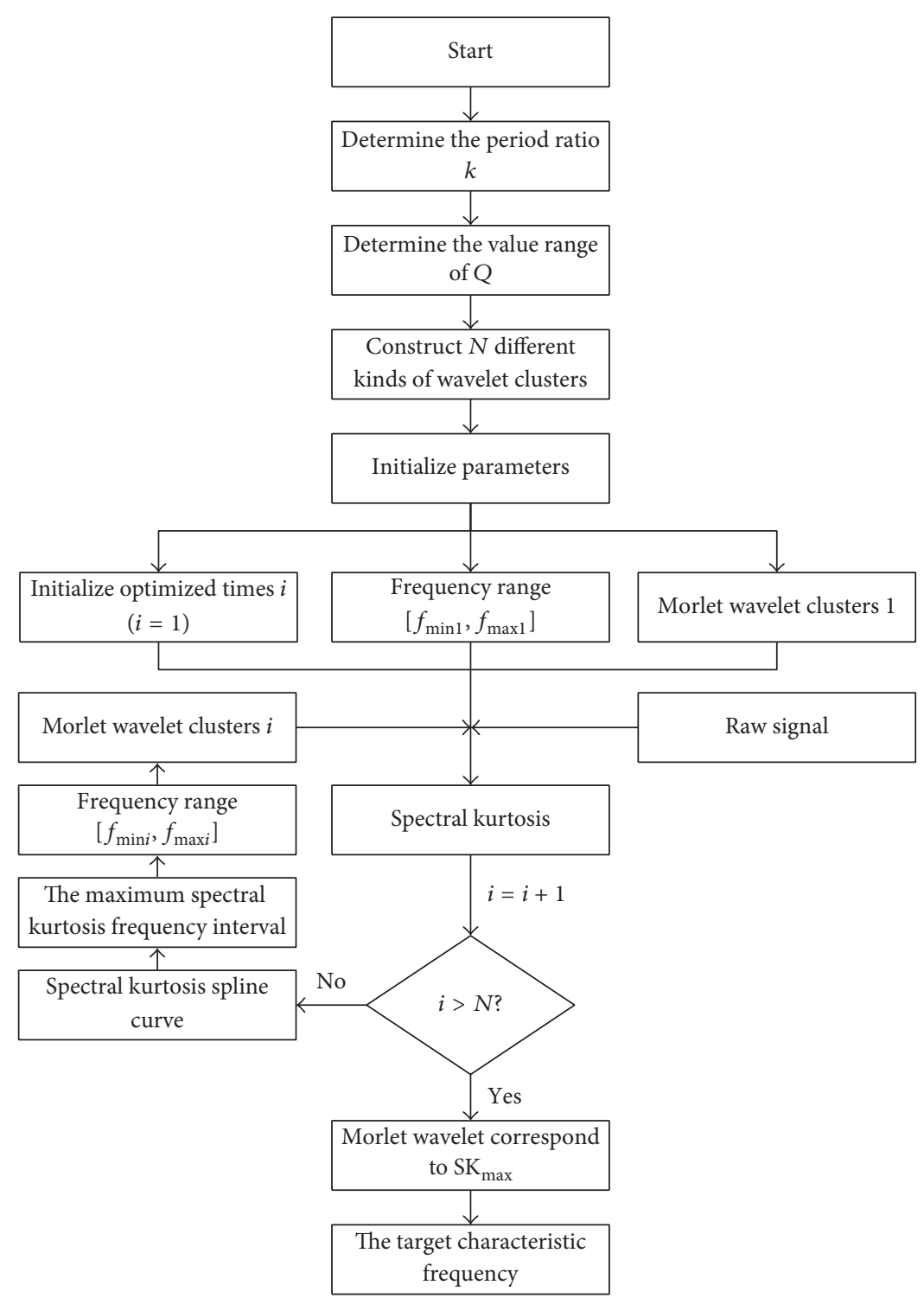

Figure 2: Program flow chart.

obtained by (16) and (17). The results are introduced into (1); then, $N$ Morlet wavelet clusters for signal processing are obtained.

When the frequency analysis range is optimized by the spline curve, the same number of filters is allocated within a smaller frequency range. As a result, the fault center frequency is more accurate and the bandwidth of the filter is smaller.

2.5. Adaptive Wavelet Method Based on Spectrum Diagnosis. Obviously, the proportion of the impact component in each filter window is different and it directly influences the spectral kurtosis of the filtering results. The former one is large means that the latter one is also large. The value of the quality factor $Q$ affects the shape of the wavelet clusters. Therefore, each quality factor corresponds to an optimization process. For a rolling element bearing, the signal components related to the rotational speed usually cannot be extracted from the timefrequency representation directly. A new proposed method is presented to solve the above problems, as shown in Figure 2:

(1) In the frequency band range containing the bearing characteristic frequencies $\left[f_{\min 1}, f_{\max 1}\right]$, it can calculate the spectral kurtosis of the filtered result and describe the spectral kurtosis by the spline curve interpolation along the frequency axis.

(2) By solving the derivation of the spline curve, the peak containing maximum spectral kurtosis and the valley on both sides of the maximum spectral kurtosis could be obtained, respectively, which is the frequency range of the next optimizing calculation $\left[f_{\min 2}, f_{\max 2}\right]$.

(3) The quality factor $Q$ is changed and the range of Morlet wavelet is reduced to $\left[f_{\min 2}, f_{\max 2}\right]$; the spectral 


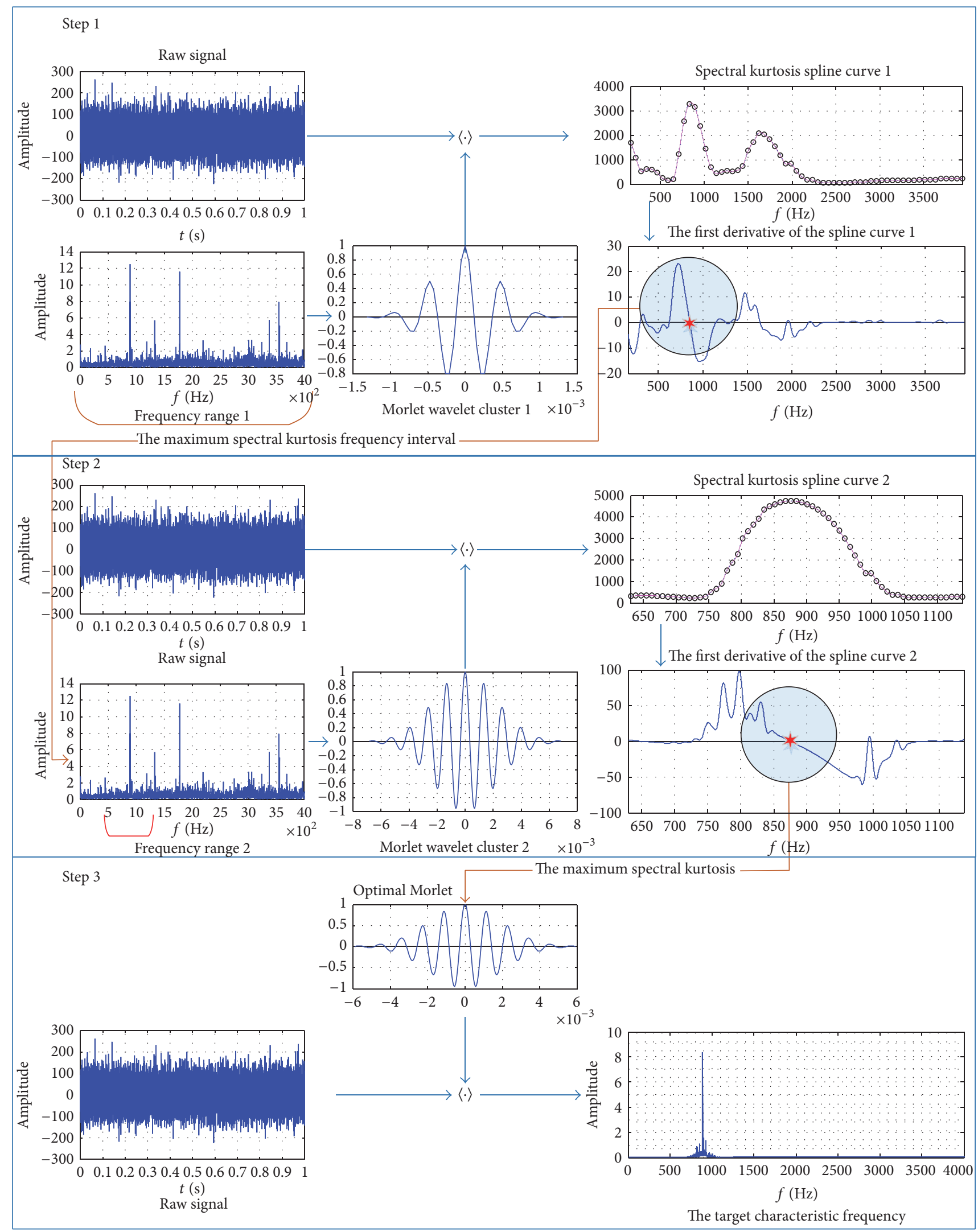

FIgURE 3: Quadratic optimization flow chart. 


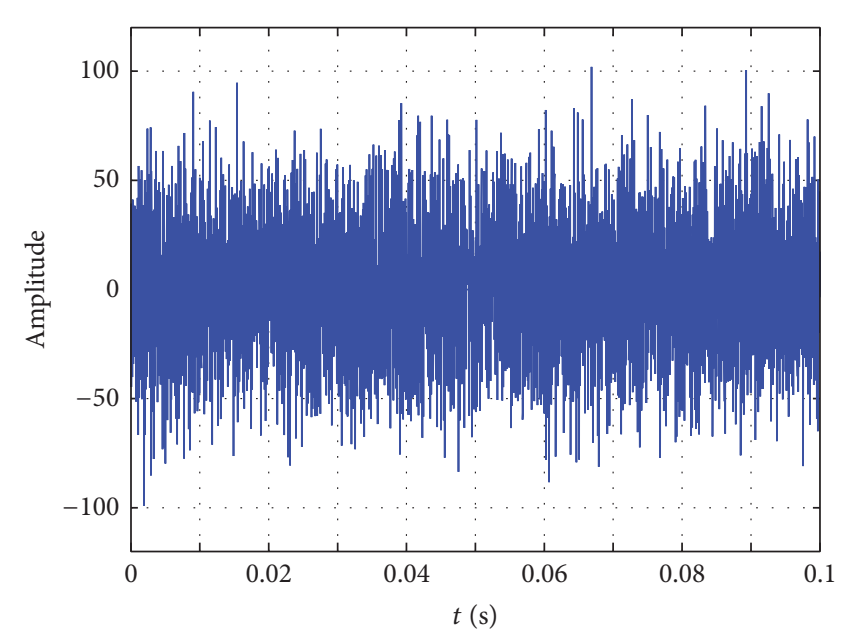

(a)

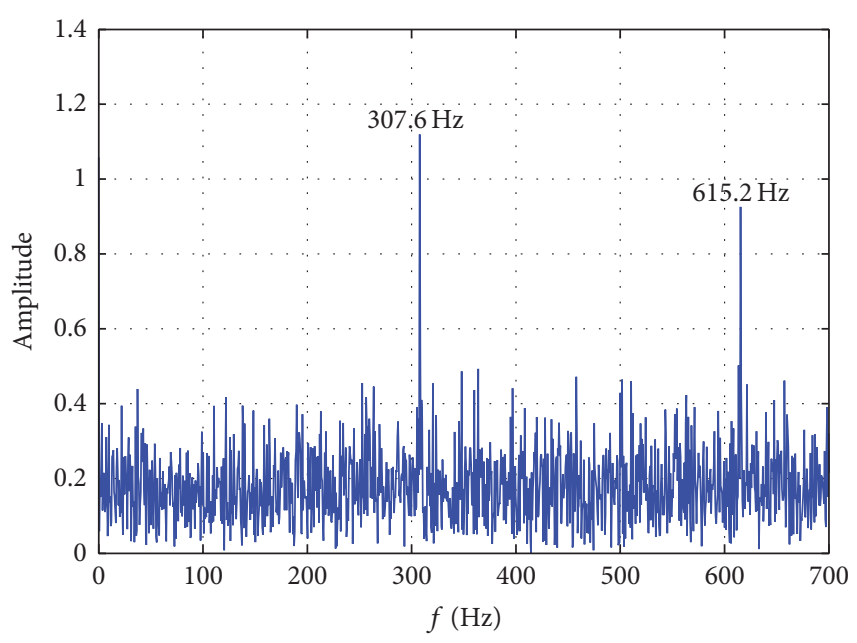

(b)

FIgURE 4: (a) Simulation signal in time domain and (b) simulation signal in frequency domain.

TABLE 1: The specific test data.

\begin{tabular}{lcccc}
\hline Parameter & Dimension of fault & Shaft rotation speed & Motor loads & Sampling frequency \\
\hline Value & 0.007 in & $29.53 \mathrm{~Hz}$ & $1 \mathrm{HP}$ & $12 \mathrm{kHz}$ \\
\hline
\end{tabular}

kurtosis of the filter bandwidths can be calculated continually. The spline curve of the spectral kurtosis can be obtained by the spline curve interpolation. However, the bandwidth of the filter is smaller than the last step.

(4) Steps (2) and (3) are repeated, until the characteristic frequency and its bandwidth are identified. The target can be achieved usually after several optimization calculations.

The calculation procedure of quadratic optimized filter is depicted iconically in Figure 3. The optimized result of Step 1 becomes the process object in Step 2. At the same time, the parameters of the Morlet wave are also adjusted. According to the result of Step 2, the fault signal was extracted in Step 3.

\section{Methods Validation}

3.1. Simulation Analysis. The validity of proposed method is verified by the bearing fault simulation signal under strong noise background. The fault simulation signal including a natural frequency of $7000 \mathrm{~Hz}$ and a fault signal frequency of $307.6 \mathrm{~Hz}$ is generated. A white Gaussian noise of $25 \mathrm{~dB}$ is added to the signal and the noise ratio is 5.49, as shown in Figure 4.

The bearing fault simulation signal is detected by the above method. First of all, the spectral kurtosis is calculated along the initial pass band. The impact frequency is $268.8 \mathrm{~Hz}$, and $\mathrm{SK}_{\max }=310.8$, as shown in Figure 5(a). Then, the impact frequency is $297.5 \mathrm{~Hz}$, and $\mathrm{SK}_{\max }=682.1$ in the frequency range narrowed in the previous step, as shown in Figure 5(b). Thirdly, the frequency range is further reduced. The impact frequency is $307 \mathrm{~Hz}$, and $\mathrm{SK}_{\max }=5652$, as shown in
TABLE 2: The bearing characteristic frequency.

\begin{tabular}{lccc}
\hline Inner ring & Outer ring & Rolling element & Cage \\
\hline $159.93 \mathrm{~Hz}$ & $105.73 \mathrm{~Hz}$ & $139.21 \mathrm{~Hz}$ & $11.76 \mathrm{~Hz}$ \\
\hline
\end{tabular}

Figure 5(c). The optimization is close to the impact frequency gradually and the bandwidth is gradually reduced at each time.

Finally, the bearing fault frequency is detected in $307.6 \mathrm{~Hz}$ and the bandwidth is about $200 \mathrm{~Hz}$ as shown in Figure 6(a). Figure 6(b) shows the result of STFT-based SK [14]. The maximum spectral kurtosis $\left(K_{\max }=0.3\right)$ is at Leave 4.5, the center frequency $f_{c}=208.33 \mathrm{~Hz}$, and the bandwidth $\mathrm{BW}=416.67 \mathrm{~Hz}$. The center frequency $f_{c}$ does not match better with impact frequency and the bandwidth BW is too wide to cover the feature frequency band precisely compared with the proposed method.

3.2. Experimental Tests. This method is verified by detecting the test data of the Case Western Reserve University Bearing Data Center [20]. In the experiment process, the bearing (SKF6205) was seeded with faults by electrodischarge machining (EDM) previously. A 0.007-inch fault was introduced at the outer raceway of faulted bearings. Then, this bearing was reinstalled into the test motor. Vibration data was recorded for motor loads of 1 horsepower and motor speeds of 1772 RPM. The specific test data is shown in Table 1.

The bearing characteristic frequency under this condition is shown in Table 2. Therefore, the initial analysis band range is designed as $[5,300] \mathrm{Hz}$. The recorded vibration signal is shown in Figure 7. 


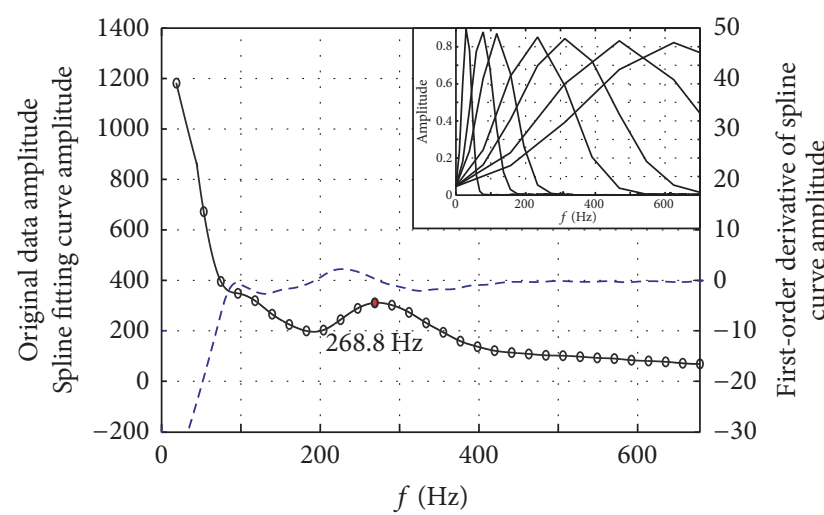

- Original data

— Spline fitting curve

- - First-order derivative of spline curve

(a)

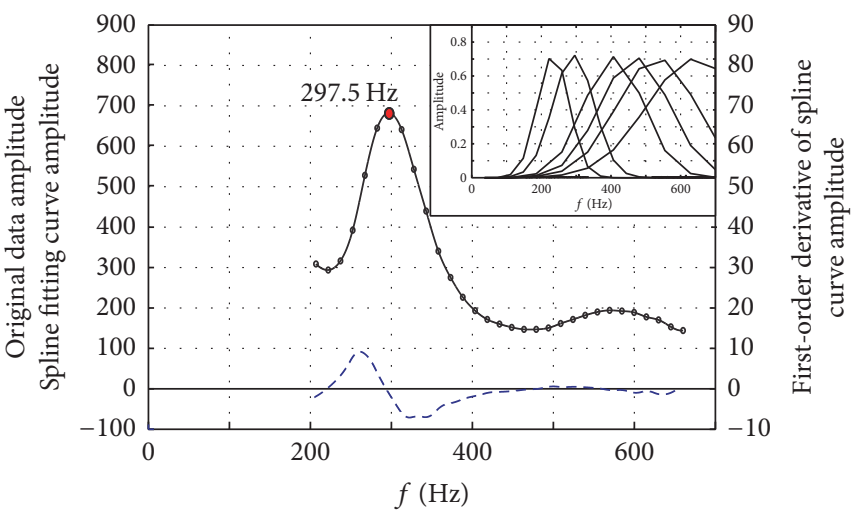

- Original data

- Spline fitting curve

- - - First-order derivative of spline curve

(b)

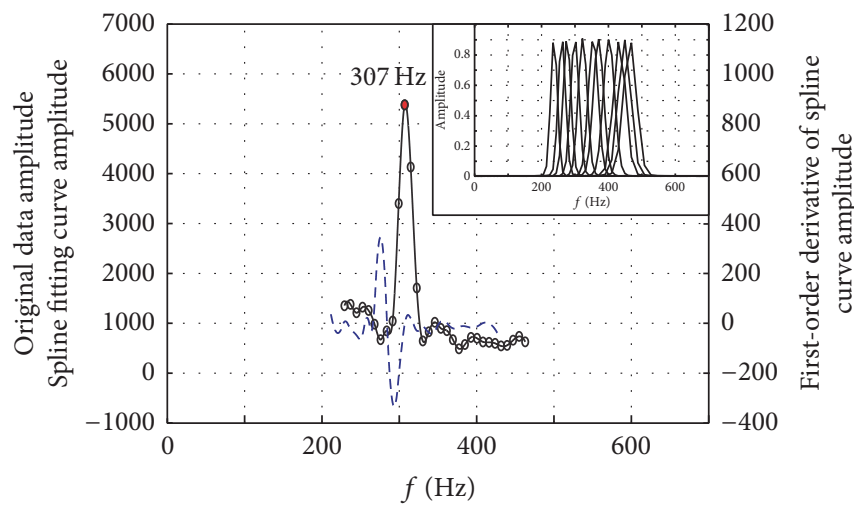

- Original data

_- Spline fitting curve

- - - First-order derivative of spline curve

(c)

FIgURE 5: (a) The first optimization result of simulation signal. (b) The second optimization result of simulation signal. (c) The third optimization result of simulation signal.

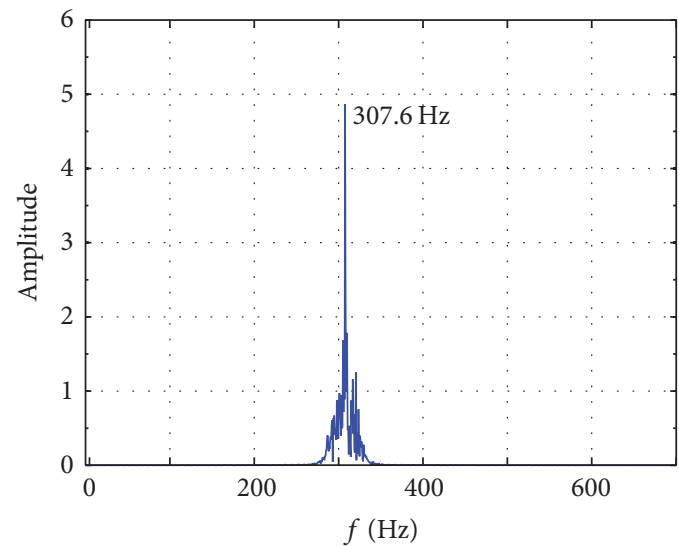

(a)

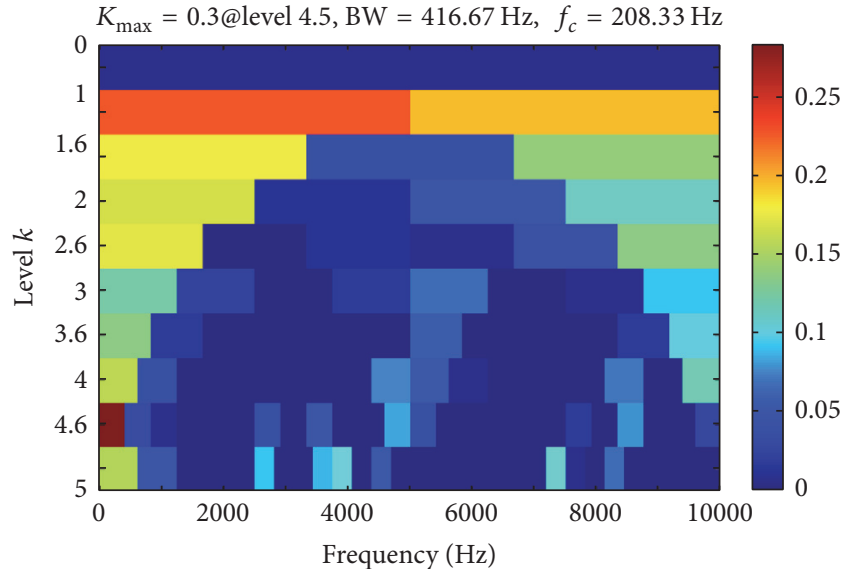

(b)

FIgURE 6: (a) Impact signal in frequency domain. (b) Fast Kurtogram of simulation signal verification. 


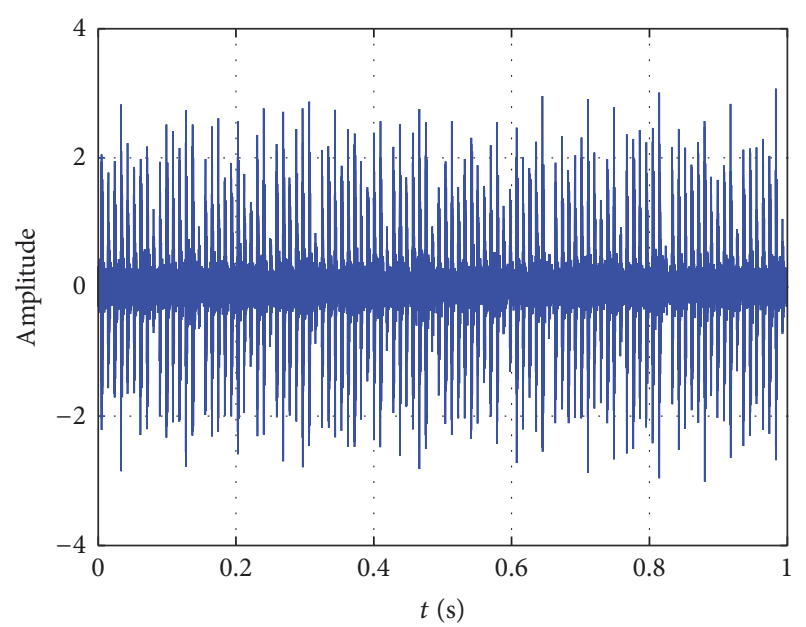

(a)

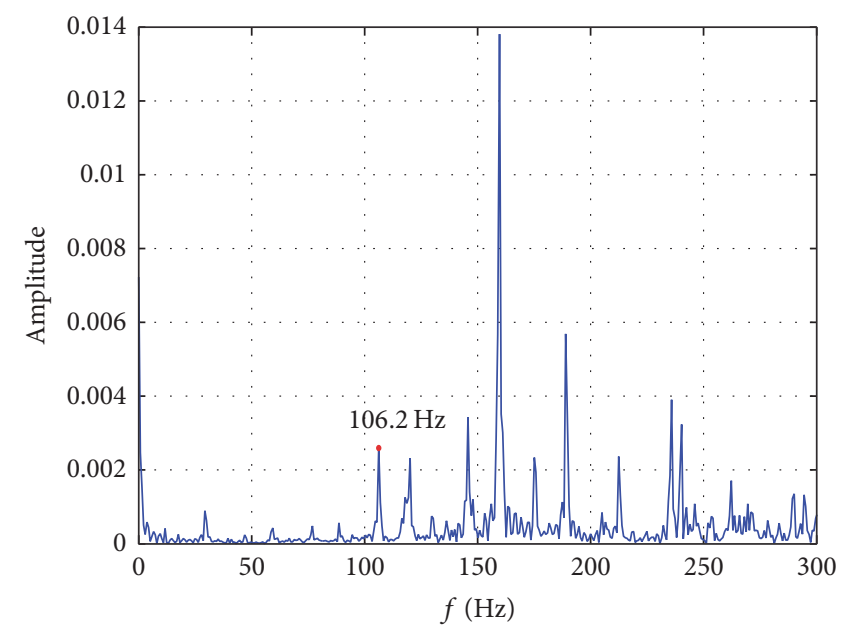

(b)

Figure 7: (a) The vibration signal in time domain. (b) The vibration signal in frequency domain.

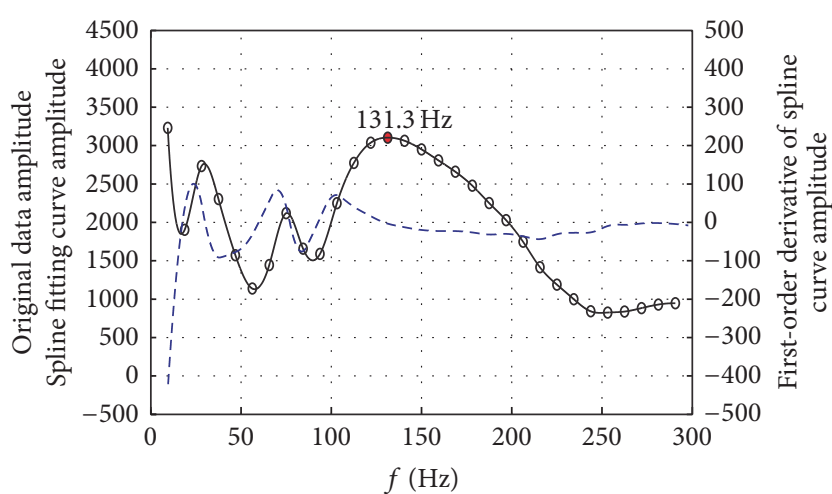

- Original data
- Spline fitting curve
_- First-order derivative of spline curve

(a)

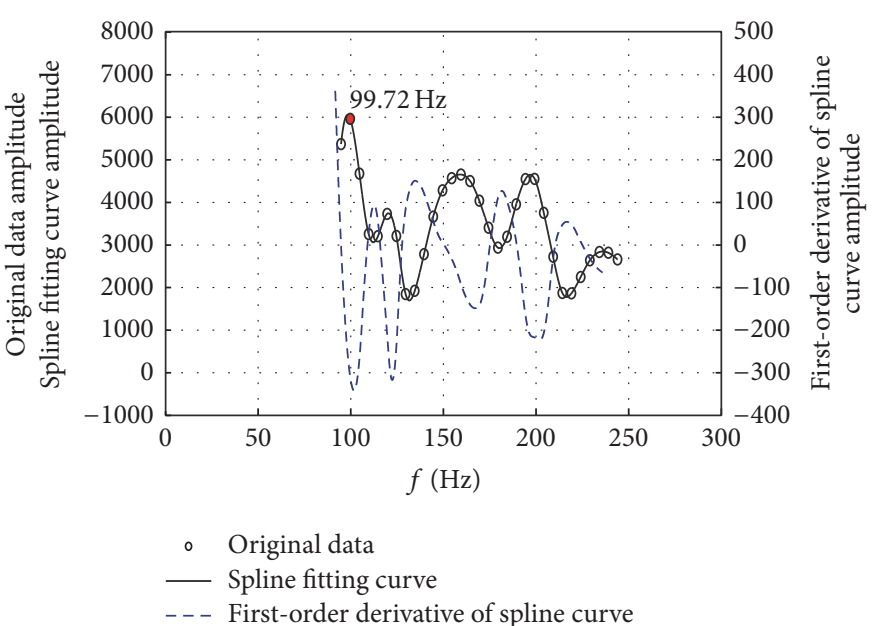

(b)

FIGURE 8: (a) The first optimization result of vibration signal. (b) The second optimization result of vibration signal.

The initial maximal spectral kurtosis and the impact frequency are 3104 and $131.3 \mathrm{~Hz}$, respectively. Then, the spectral kurtosis is calculated in the optimized frequency band, and it can be extracted that the impact frequency is $99.72 \mathrm{~Hz}, \mathrm{SK}_{\max }=5959$ in Figure 8.

The fault frequency is $106.2 \mathrm{~Hz}$ and the fault signal can be identified clearly in the frequency domain and time domain as shown in Figure 9.

\section{Conclusion}

The method was based on the similarity between Morlet wavelet and faulty impulse signal. The spectral kurtosis maximum principle was employed into wavelet spectrum detection method for rolling element bearing fault signal as a criterion. At the initial filtering of the raw signal, the frequency range of the fault signal was found by the spline curve interpolation and its derivation. Different wavelet clusters that produced by the quality factor $Q$ could optimize the searching range gradually and reduce the filter window width. The fault diagnosis ability of rolling bearing was improved. In conclusion, according to the treatment of the simulation signal and the bearing experimental test signal by the proposed method, it showed that the proposed method recognized the fault signal accurately and had better bearing fault time-frequency recognition feature.

\section{Competing Interests}

The authors declare that they have no competing interests. 


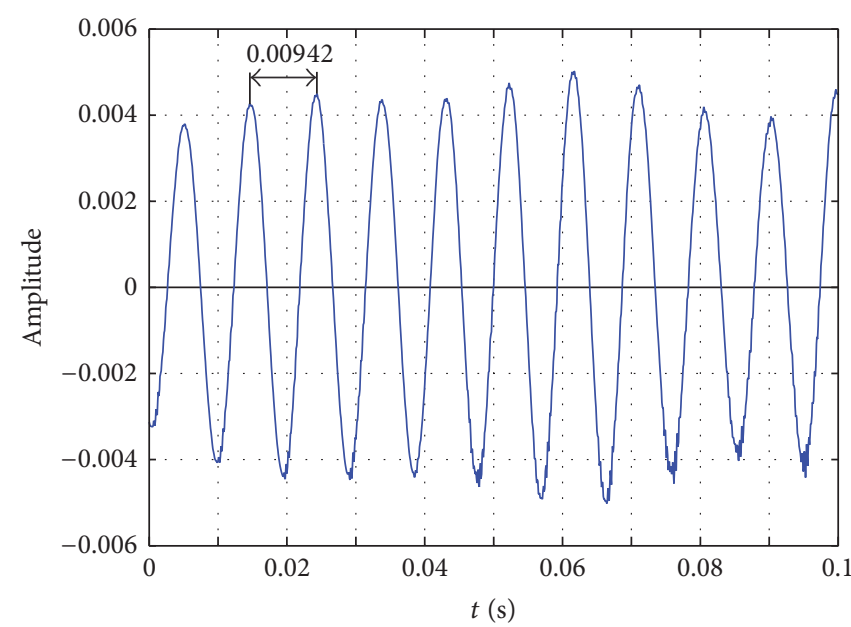

(a)

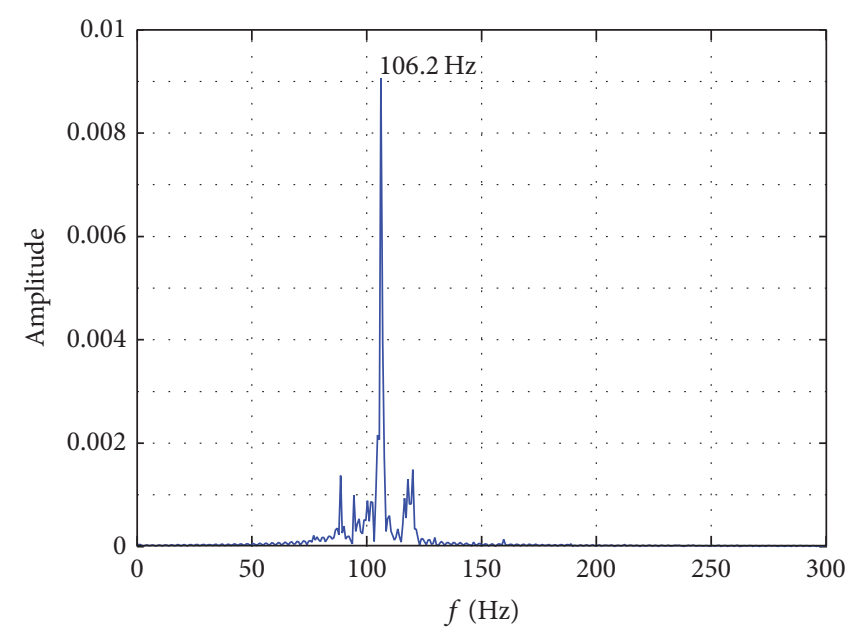

(b)

Figure 9: (a) Bearing fault signal in frequency domain. (b) Bearing fault signal in time domain.

\section{Acknowledgments}

This research is supported by National Key Basic Research Program of China (973 Program) under Grant no. 2013CB632305 and the Foundation for Innovative Research Groups of the National Natural Science Foundation of China under Grant no. 51521003.

\section{References}

[1] I. El-Thalji and E. Jantunen, "Fault analysis of the wear fault development in rolling bearings," Engineering Failure Analysis, vol. 57, pp. 470-482, 2015.

[2] S. Prabhakar, A. S. Sekhar, and A. R. Mohanty, "Detection and monitoring of cracks in a rotor-bearing system using wavelet transforms," Mechanical Systems and Signal Processing, vol. 15, no. 2, pp. 447-450, 2001.

[3] Y. Lei, J. Lin, Z. He, and M. J. Zuo, "A review on empirical mode decomposition in fault diagnosis of rotating machinery," Mechanical Systems and Signal Processing, vol. 35, no. 1-2, pp. 108-126, 2013.

[4] N. Tandon and A. Choudhury, "A review of vibration and acoustic measurement methods for the detection of defects in rolling element bearings," Tribology International, vol. 32, no. 8, pp. 469-480, 1999.

[5] P. K. Kankar, S. C. Sharma, and S. P. Harsha, "Fault diagnosis of ball bearings using continuous wavelet transform," Applied Soft Computing, vol. 11, no. 2, pp. 2300-2312, 2011.

[6] C. T. Yiakopoulos and I. A. Antoniadis, "Wavelet based demodulation of vibration signals generated by defects in rolling element bearings," Shock and Vibration, vol. 9, no. 6, pp. 293306, 2002.

[7] R. B. Randall and J. Antoni, "Rolling element bearing diagnostics-a tutorial," Mechanical Systems and Signal Processing, vol. 25, no. 2, pp. 485-520, 2011.

[8] X. Zhang, J. Kang, L. Xiao, J. Zhao, and H. Teng, "A new improved Kurtogram and its application to bearing fault diagnosis," Shock and Vibration, vol. 2015, Article ID 385412, 22 pages, 2015.
[9] H. Liu, W. Huang, S. Wang, and Z. Zhu, "Adaptive spectral kurtosis filtering based on Morlet wavelet and its application for signal transients detection," Signal Processing, vol. 96, pp. 118124, 2014.

[10] R. F. Dwyer, "A technique for improving detection and estimation of signals contaminated by under ice noise," Journal of the Acoustical Society of America, vol. 74, no. 1, pp. 124-130, 1983.

[11] R. F. Dwyer, "Use of the kurtosis statistic in the frequency domain as an aid in detecting random signals," IEEE Journal of Oceanic Engineering, vol. 9, no. 2, pp. 85-92, 1984.

[12] R. F. Dwyer, "Detection of non-Gaussian signals by frequency domain kurtosis estimation," in Proceedings of the IEEE International Conference on Acoustics, Speech and Signal Processing (ICASSP '83), pp. 607-610, Boston, Mass, USA, 1983.

[13] J. Antoni and R. B. Randall, "The spectral kurtosis: application to the vibratory surveillance and diagnostics of rotating machines," Mechanical Systems and Signal Processing, vol. 20, no. 2, pp. 308-331, 2006.

[14] J. Antoni, "Fast computation of the kurtogram for the detection of transient faults," Mechanical Systems and Signal Processing, vol. 21, no. 1, pp. 108-124, 2007.

[15] K. Ding, Z.-D. Huang, and H.-B. Lin, "A weak fault diagnosis method for rolling element bearings based on Morlet wavelet and spectral kurtosis," Journal of Vibration Engineering, vol. 27, no. 1, pp. 128-135, 2014.

[16] N. Sawalhi and R. B. Randall, "Spectral kurtosis optimization for rolling element bearings," in Proceedings of the 8th International Symposium on Signal Processing and Its Applications (ISSPA '05), vol. 2, pp. 839-842, IEEE, August 2005.

[17] Q. Li and C. Wang, Numerical Analysis, Tsinghua University Press, Beijing, China, 4th edition, 2001.

[18] J. Antoni, “The spectral kurtosis: a useful tool for characterising non-stationary signals," Mechanical Systems and Signal Processing, vol. 20, no. 2, pp. 282-307, 2006.

[19] Y. Guo, H. Zheng, Y. Gao, and T. Wu, "The spectral envelope of rolling bearing analysis based on kurtosis," Journal of Vibration, Measurement \& Diagnosis, no. 4, pp. 517-539, 2011.

[20] W. A. Smith and R. B. Randall, "Rolling element bearing diagnostics using the Case Western Reserve University data: a benchmark study," Mechanical Systems and Signal Processing, vol. 64-65, pp. 100-131, 2015. 


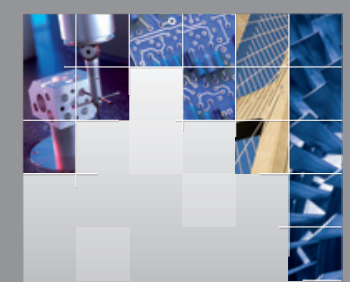

\section{Enfincering}
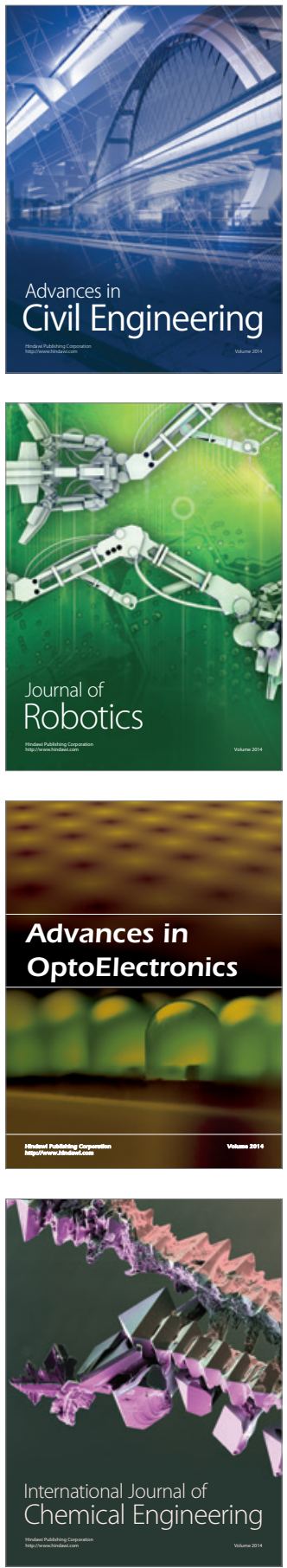

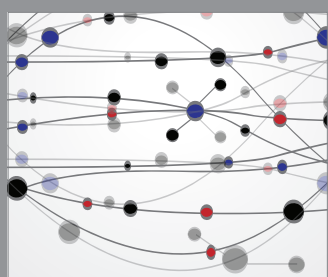

The Scientific World Journal

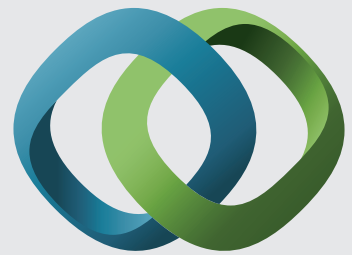

\section{Hindawi}

Submit your manuscripts at

https://www.hindawi.com
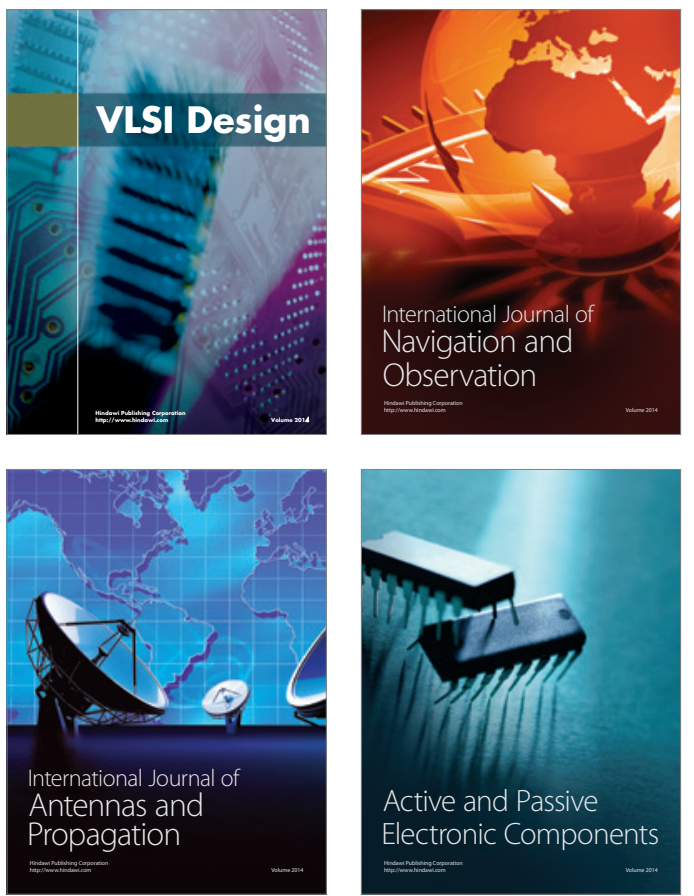
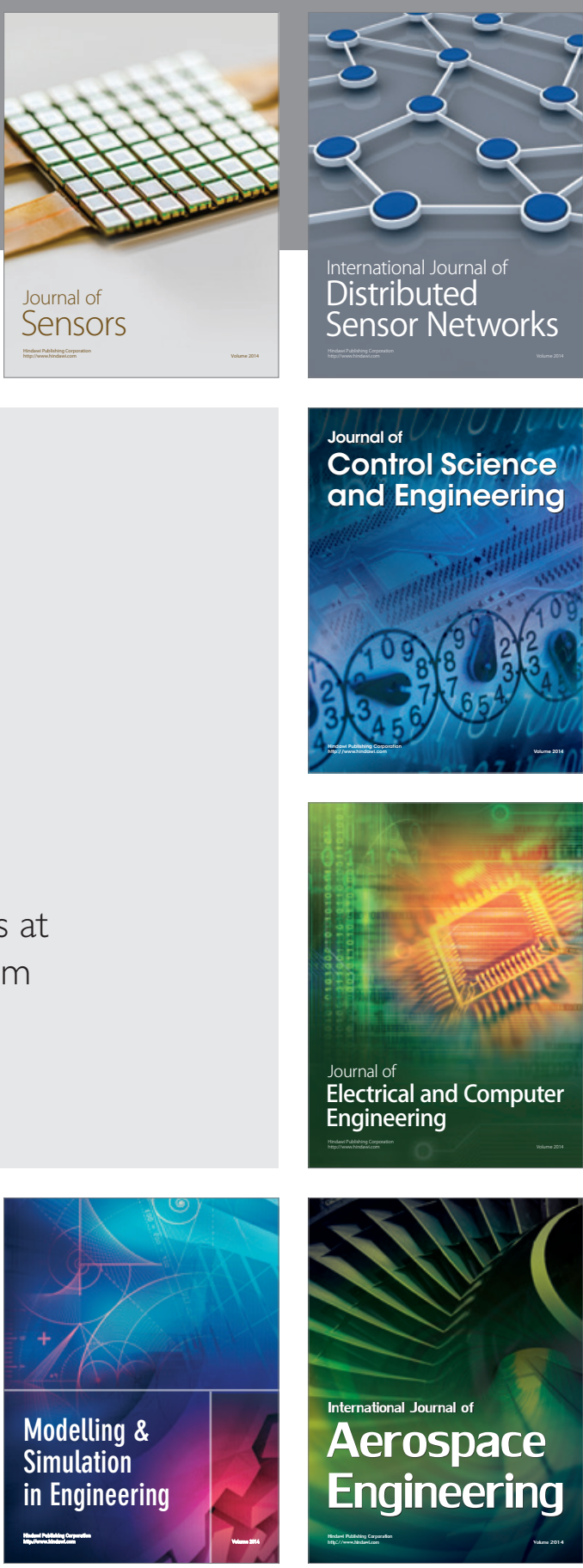

International Journal of

Distributed

Sensor Networks

$-$

Joumal of

Control Science

and Engineering
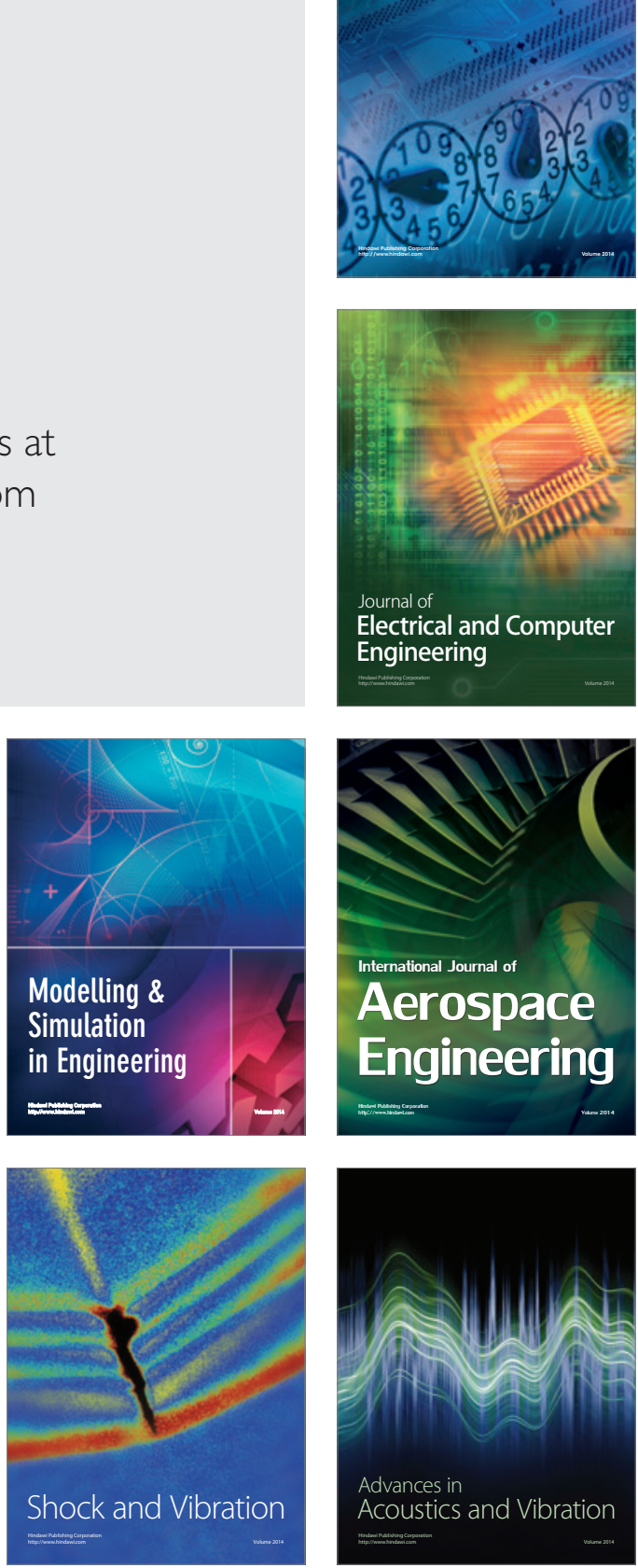